\title{
Reduplicación en la nominalización de juegos de niños en el quechua ayacuchano
}

\section{Reduplication in the Nominalization of Children's Games in Ayacucho Quechua}

\author{
Fernando Aarón Torres Castillo \\ Universidad Nacional Mayor de San Marcos, Lima, Perú \\ fernando.torres2@unmsm.edu.pe \\ Ana Priscila Ugarte de la Fuente \\ Universidad Nacional Mayor de San Marcos, Lima, Perú \\ ana.ugarte@unmsm.edu.pe
}

\begin{abstract}
Resumen
El proceso de reduplicación es un fenómeno lingüístico que está presente en muchas lenguas y cumple diferentes funciones semánticas. A veces, la reduplicación es utilizada para expresar pluralidad en categorías nominales y, en otras, para deverbalizar un verbo. En el presente estudio, se describe la reduplicación que existe en el proceso de nominalización de los juegos cotidianos de niños y niñas de Ayacucho. Estos nombres impuestos por los niños presentan formaciones diferentes y recursivas debido a las funciones semánticas.
\end{abstract}

Palabras clave: Reduplicación; juegos infantiles; nominalización.

\begin{abstract}
Reduplication process is a linguistic phenomenon that is present in many languages and has different semantic functions. Sometimes, reduplication is used to express the plurality in a language, meanwhile in others its function is to deverbalize a verb. In this study, we describe the reduplication given in a nominalization process in Ayacucho Quechua for children games. These names are imposed by children and have different recursive constructions due to semantic functions.
\end{abstract}

Keywords: Reduplication; Quechua; children games; nominalization. 


\section{Introducción}

La reduplicación no es un proceso nuevo, ya que está presente en distintas lenguas; un ejemplo de ello es la reduplicación parcial presente en el latín: mordēre 'hincar el diente' > momordisse 'haber hincado el diente'. Este fenómeno es muy productivo y puede derivar en la formación de sustantivos, adjetivos, adverbios e, incluso, verbos. Sin embargo, son pocas las investigaciones del fenómeno de la reduplicación en lenguas indígenas del Perú, tales como el quechua, el aymara, el cauqui, el jaqaru, entre otros. Soto (2006) define la reduplicación como la «repetición de raíces o morfemas completos o parte de ellos con la finalidad de expresar un significado nuevo», como en el ejemplo hatun hatun 'enorme'. Asimismo, para el caso del jaqaru, Escobar (2017) indica que esta lengua presenta el fenómeno de reduplicación con "características morfofonémicas distintas [al quechua y aymara] y, más bien, tiene semejanza con algunas de las lenguas del mundo [como el euskera o el mapuche] que han puesto en práctica también este fenómeno de formación léxica».

En el quechua de Ayacucho se evidencia el uso de la reduplicación, por lo que en esta investigación se presenta, en primer lugar, el marco conceptual con el que se va a explicar el fenómeno de la reduplicación en la nominalización de los juegos de los niños en quechua ayacuchano, luego se describe el fenómeno de la reduplicación a partir de la información obtenida respecto de otras lenguas; después se presenta un marco general en la lengua quechua, tanto los tipos de reduplicación semántica que existen en la bibliografía actual, como la forma morfológica en la que se expresan; más adelante, se muestra el corpus organizado para el análisis. Finalmente, se plantean conclusiones y recomendaciones respecto del tema investigado, con lo que se espera contribuir a los antecedentes de estudio.

A todo esto, el presente estudio responde a las siguientes problemáticas:

a. ¿Qué funciones semánticas se manifiestan para que la nominalización de los juegos de los niños ayacuchanos se reduplique?

b. ¿Cómo se relacionan los procesos morfológicos en la reduplicación de las nominalizaciones de los juegos de los niños en Ayacucho?

\section{MARCO CONCEPTUAL}

Con el fin de explicar la reduplicación en la nominalización en quechua de los juegos de los niños en la región de Ayacucho, se considera el trabajo realizado por Escobar Zapata (2013) «La reduplicación en el idioma jaqaru, interacción de la 
fonología y morfología». A partir del artículo publicado por la autora, se extrajo los rasgos semánticos que utilizó para responder a la problemática de cómo se configura la reduplicación en jaqaru. Por lo que se ha realizado el siguiente esquema para evidenciar los rasgos semánticos a utilizar en el presente artículo.

\begin{tabular}{l|l}
\hline [oscilación] & Se describe como cambio de lugar de forma repetitiva. \\
\hline [cantidad] & Indica suma de un aspecto, aumento de la interacción. \\
\hline [frecuencia] & $\begin{array}{l}\text { Se describe como la idea de continuidad de una situación } \\
\text { temporal de forma periódica. }\end{array}$ \\
\hline $\begin{array}{l}\text { [en varios puntos o } \\
\text { espacios] }\end{array}$ & $\begin{array}{l}\text { También llamado fragmentación o división de la unidad en } \\
\text { partes, idea de distribución en diferentes lugares o en un } \\
\text { mismo lugar concordante. }\end{array}$ \\
\hline [rapidez de la acción] & $\begin{array}{l}\text { Indica pluralidad como marca de la continuidad de una } \\
\text { acción, similar a un intensificador. }\end{array}$ \\
\hline
\end{tabular}

\section{La reduplicación en algunas lenguas del mundo}

Hualde (2001, p. 350) muestra un ejemplo muy interesante en el español en contacto con las lenguas mayas en el sur de México, en la zona de Yucatán y Chiapas, y en Guatemala, de los estudios sobre el español en estas regiones, presenta lo siguiente: «La reduplicación de -ísimo en -isísimo: riquisisísimo, pobrisisísimo».

En el trabajo de Martínez (1994, p. 163) se presenta el fenómeno de la reduplicación en raíces nominales de la lengua yaqui, tanto reduplicación primaria como reduplicación secundaria:

Red. Primaria tipo cv:

ká.ba (huevo) ka.-ká.ba (poner huevos)

Red. Secundaria tipo cvc:

ká.ba (huevo) kak.-ká.ba (poner huevos de vez en cuando)

En los ejemplos anteriores se pueden observar las diferencias a nivel semántico entre la reduplicación primaria (Cv) y la secundaria (CVC). Se podría decir que en este caso la reduplicación secundaria tiene una función que indica [frecuencia] a nivel temporal.

Armendáriz (2007) indica que los nombres en guarijío generalmente no distinguen la pluralidad, excepto en algunos sustantivos que refieren a personas, relación de parentesco y en algunos préstamos del español que también denotan entidades humanas. Se muestra un ejemplo por cada patrón, en Armendáriz (ibídem: 26). 


$\begin{array}{llll}\text { CV- } & \text { wocí } & \text { wo-wocí } & \text { 'abuelo' } \\ \text { CV'- } & \text { pací } & \text { pa'-pací } & \text { 'hermano mayor' } \\ \text { Cvh- } & \text { piní } & \text { pih-piní } & \text { 'hermana menor' } \\ \text { CV'y acento } & \text { suhkitúme } & \text { su'-súgirume } & \text { 'brujo' }\end{array}$

Para la lengua guarijío, la función que expresa la reduplicación es de [parentesco], la cual es muy productiva en esta lengua.

En la lengua tepehuano del sur, los nombres pueden pluralizarse a través de una sílaba reduplicada prefijada a la raíz y no es fácil predecir si la vocal reduplicada será corta o larga. Guerrero (2014, p. 10) señala que como en otras lenguas de la familia Yaqui, a la que pertenece el tepehuano, con frecuencia la raíz nominal presenta una serie de «ajustes» al reduplicarse: apócope, síncope, reducción vocálica, truncación y resilabificación.

$\begin{array}{lll}\text { juk } & \text { ju-juk } & \text { 'pinos' } \\ \text { jo' } & \text { ja-jo' } & \text { 'pieles' } \\ \text { bhan } & \text { bhaa-bhan } & \text { 'coyotes' } \\ \text { bai } & \text { báa-bai } & \text { 'colas' }\end{array}$

En este caso, la reduplicación desempeña una función de [cantidad]. La lengua configura la reduplicación de la primera sílaba y en algunos casos se alarga la vocal del segmento reduplicado.

Al igual que la lengua tepehuana del sur, un caso más relacionado con la presente investigación se observa en la lengua aimara, donde la reduplicación también se manifiesta con la función de [cantidad].

Esto se puede observar en la siguiente tabla:

Tabla 1.

\begin{tabular}{l|l}
\hline $\begin{array}{l}\text { collo } \\
\text { collo collo }\end{array}$ & $\begin{array}{l}\text { n. cerro } \\
\text { n. cordillera }\end{array}$ \\
\hline coca & n. árbol \\
coca coca & n. arboleda \\
\hline cala & n. piedra \\
cala cala & n. pedregal
\end{tabular}

Extraído de El Jaqaru, último relicto aru en los Andes Centrales (2017:76), de Emérita Escobar. 


\section{La reduplicación en el quechua}

En este apartado se presenta el fenómeno de la reduplicación en diferentes variedades de la lengua quechua. David Weber, en Gramática del quechua de Huallaga (1996), presenta distintos tipos de reduplicación para la variedad del Huallaga, de los cuales algunos se muestran a continuación:

\subsection{RED dentro de ítems léxicos}

Weber (1996, p. 417) muestra algunos de los elementos reduplicados que son el resultado de la posposición de ítems léxicos, por lo que se tiene dos palabras de la misma naturaleza con un significado diferente al original.

Tabla 2.

\begin{tabular}{l|l}
\hline $\begin{array}{l}\text { macha } \\
\text { macha macha }\end{array}$ & $\begin{array}{l}\text { loc v. estar borracho } \\
\text { n. baya silvestre (se dice que produce mareos) }\end{array}$ \\
\hline $\begin{array}{l}\text { paka } \\
\text { paka paka }\end{array}$ & $\begin{array}{l}\text { v. esconder } \\
\text { n. especie de ave }\end{array}$
\end{tabular}

Basado en Una gramática del quechua de Huallaga (Huánuco) (1996, p. 417), de David Weber.

En los ejemplos presentados, la reduplicación es de toda una raíz; como se observa, una locución verbal deriva en un nombre, como sucede con el verbo en el segundo ejemplo.

\subsection{RED de palabras simples}

Según Weber (1996, p. 418), las palabras no-verbales pueden reduplicarse sin cambiar la naturaleza de la raíz; asimismo, la raíz nominal puede reduplicarse con el sufijo /-n/ o con algún sufijo de caso. 
Tabla 3.

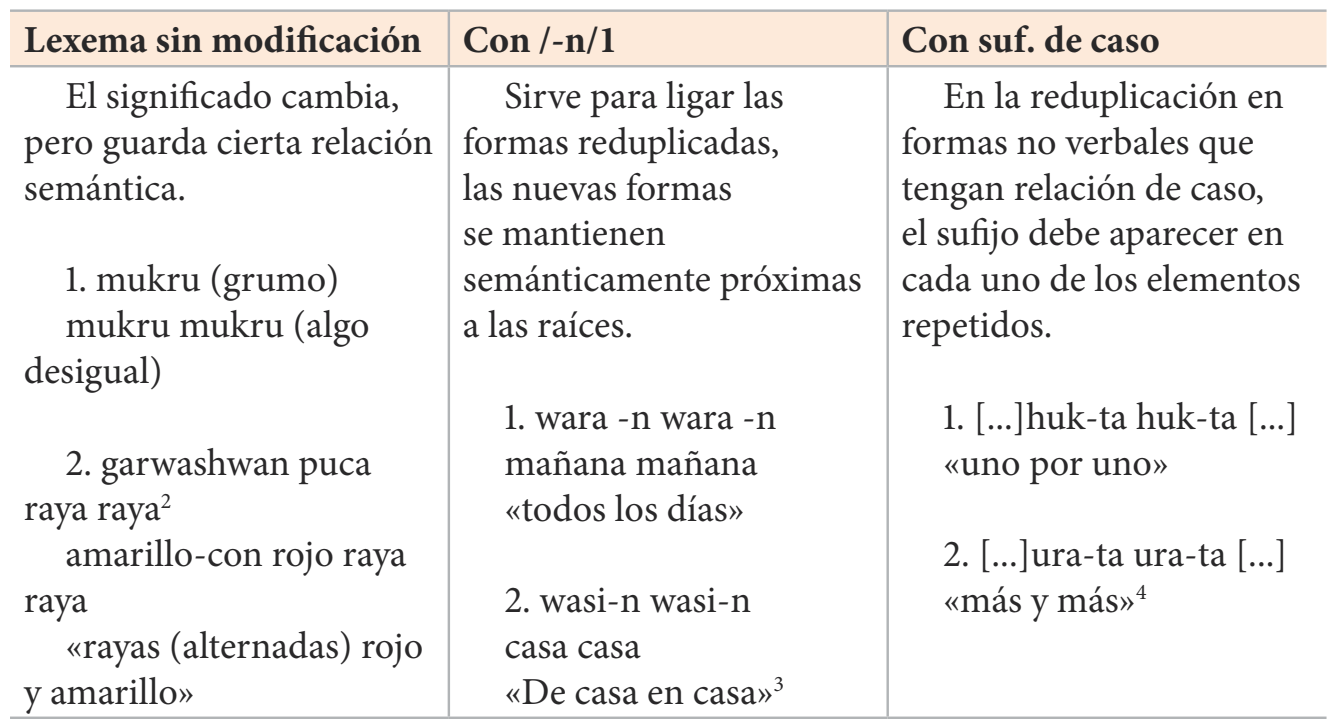

Basado en Una Gramática del quechua de Huallaga (Huánuco) (1996, p. 418), de David Weber.

\subsection{RED adverbio verbo}

Weber $(1996,421)$ señala que una de las formas más comunes de reduplicar un verbo es convertir la primera aparición en una cláusula adverbial que esté subordinada a la segunda. Existen casos con los sufijos /-r/ y /-y/ /-pa/. A continuación se muestra el sufijo - $r$ que actúa como un adverbializador (sujeto idéntico).

1 No tiene función significativa.

2 Raya viene del español raya 'línea'.

3 Un caso muy especial para este tipo de reduplicación se manifiesta en el siguiente ejemplo: Wasiquicunata ricachimay maygan maygantataj $\rightarrow$ 'Muéstrame cuáles son tus casas' (cuando las casas están dispersas por el pueblo). Claramente, el sufijo - $n$ en la reduplicación no solo se manifiesta de manera innecesaria, es probable que en este tipo de reduplicación se exprese la función de [en varios puntos o espacios].

4 Véase Gramática del quechua de Huallaga (1996, p. 421), de David Weber. 
Tabla 4. Formas del verbo -r

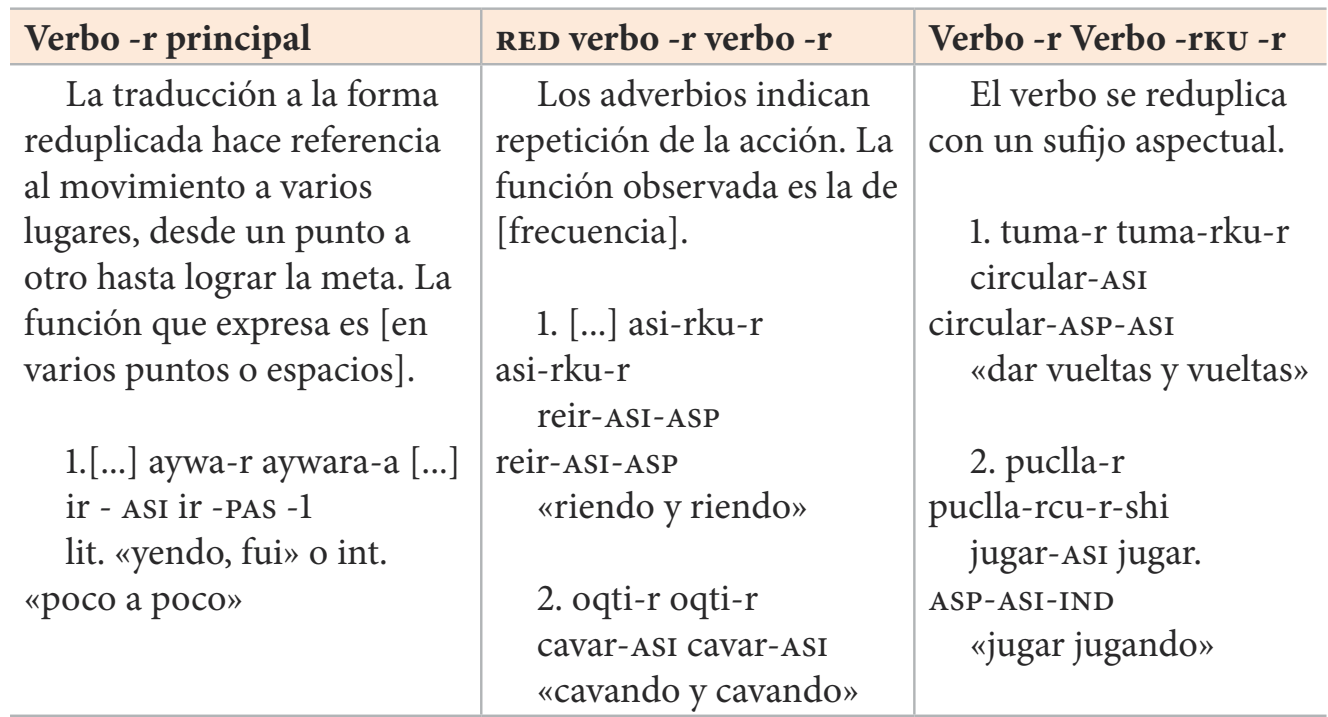

Basado en Una gramática del quechua de Huallaga (Huánuco) (1996, p. 42I), de David Weber.

Para la forma compuesta verbo/-ypa/verbo, se cuenta con el siguiente ejemplo de Weber (1996, p. 424):

\author{
«ikchu- 'suspiro profundo' \\ icchuypa icchur ikchu-y-pa ikchu-r 'sollozando'»
}

Ese sufijo /-ypa/ presenta conjuntamente con el fenómeno de la reduplicación expresa la función de [intensidad], donde el suspirar profundo constantemente o con mayor grado en la acción, se manifiesta como algo más que solo suspirar; esto sería sollozar, dado que tiene un valor más intenso.

\title{
4.4 RED de cláusulas adverbiales ${ }^{5}$
}

Weber (1996, p. 425) indica que este es un caso raro, ya que el fenómeno de la reduplicación se manifiesta de forma compleja. En el ejemplo, el segmento que se reduplica es /horqurkur kutirkachir/, pues este contiene una cláusula adverbial. 
Tabla 5.

\begin{tabular}{|c|c|}
\hline horqu-rku-r kuti-rku-chi-r & horqu-rku-r kuti-rku-chi-r \\
\hline sacar-ASP-ASI regresar-ASP-CAU-ASI & sacar-ASP-ASI regresar-ASP-CAU-ASI \\
\hline sacó repentinamente [...] y volvió a & \\
\hline
\end{tabular}

Las funciones que se observaron en este tipo de reduplicación son las de [rapidez de la acción] y [oscilación].

\subsection{RED con radicales verbales incompletos}

Weber (1996, p. 425) señala que se reduplican los verbos de forma incompleta, como es el caso de los verbos rusa-y y achikya.

Tabla 6.

\begin{tabular}{l|l}
\hline Rusachi rusachi-yllapa awlli-nchi & nina-naw achikya achikya-r aywa-n \\
\hline cruzar-CAU cruzar-CAU-ADvsI urdir-2p & llama-sım brillar brillar-ASI ir-3p \\
\hline $\begin{array}{l}\text { «Urdimos cruzando (el hilo) de acá } \\
\text { para allá» }\end{array}$ & «va desprendiendo luz como una llama» \\
\hline
\end{tabular}

Basado en Una gramática del quechua de Huallaga (Huánuco) (1996, p. 425), de David Weber.

Se observa que en el primer caso se manifiesta la función de [frecuencia] y [en varios puntos o espacios]; mientras que en el segundo sólo se presenta la función de [frecuencia], al igual que en el anterior, pero además la de [intensidad].

\subsection{RED verbo verbo}

Weber (1996, p. 426) señala que un verbo finito también puede reduplicarse, como en el caso del verbo kacha. 
Tabla 7.

wata-pti-n-si kacha-ka-sha kacha-ka-sha amarrar-ASD-3p-NE soltar-PSV-3PRF soltar-PSV-3PRF

«Aunque lo amarraban, una y otra vez se soltaba»

La función que se presenta aquí es la de [frecuencia].

\subsection{RED infinitivo infinitivo}

Como su mismo nombre indica, se reduplican dos verbos infinitivos, por ello, Weber (1996, p. 427) señala el siguiente ejemplo:

«kanta-y-ta kanta-y-ta yacha-ku-nki

'tú sabes cantarlos vez tras vez'»

Al igual que en el caso anterior, se manifiesta la función de [frecuencia].

Como lo afirma Escobar (2017, p. 76) la reduplicación en el quechua «es productiva y es una de las características de la formación léxica, sin embargo, el esquema para repetir las raíces difiere del observado en el jaqaru o aimara central».

\section{Metodología}

Para la presente investigación, los datos fueron obtenidos de los niños que jugaban en diversas partes en los pueblos cercanos a Huanta, en el departamento de Ayacucho, específicamente de las escuelas de formación en Educación Intercultural Bilingüe, donde los docentes son bilingües y la participación de los padres es activa ${ }^{6}$.

La intervención de los investigadores fue a partir de la observación y participación, se captó los nombres de los juegos mientras que los niños jugaban, para luego proceder a preguntarles el funcionamiento de cada uno de ellos, y el porqué del nombre. 


\section{Sobre el corpus}

\subsection{Tabulación del corpus}

En la siguiente Tabla, se indican los juegos encontrados:

Tabla 8. Verbos recopilados

\begin{tabular}{l|l}
\hline chilaq chilaq & También llamado el juego del zorro y la gallina. \\
\hline volca volcachi & $\begin{array}{l}\text { Juego donde los carros de los participantes son desplazados por } \\
\text { la polea donde cruzan. }\end{array}$ \\
\hline paka paka & También llamado las escondidas. \\
\hline chapa chapa & También llamado las chapadas. \\
\hline pis pis & También llamado yases. \\
\hline kallampi & Para este juego se utiliza una barra de madera y una llanta. \\
\hline taka & Juego del trompo (chantar). \\
\hline daño & Juego de las canicas. \\
\hline
\end{tabular}

\subsection{Análisis del corpus}

Para el análisis, los juegos se ha dividido en dos grupos: el primero es el de juegos reduplicados (JR), y el segundo, juegos no reduplicados (JNR). Líneas más abajo se presenta el análisis lingüístico y los aspectos culturales que intervienen en la conceptualización de las nominalizaciones.

\subsubsection{Palabras reduplicadas con sufijos}

Tabla 9. Separación de los JR y los JNR

\begin{tabular}{l|l}
\hline Juegos Reduplicados (JR) & Juegos No Reduplicados (JNR) \\
\hline volca volcachi & kallampi \\
\hline
\end{tabular}

La denominación del JR volca volcachi procede del verbo transitivo castellano volcar. Además de la reduplicación del verbo, se observa una sufijación quechua. El sufijo es /-chi/, que actúa como causativo, el cual es deverbalizador, ya que es 
un sufijo derivador. En castellano, el verbo sufijado se puede traducir como 'hacer que otro haga algo', tal como lo señala Zariquiey (2008, p. 171) en el texto Qayna, Kunan, Paqarin / Una introducción práctica al quechua chanca.

Tabla 10. Segmentación morfológica

\begin{tabular}{l|l|l}
\hline volca & volca & -chi \\
\hline verbo & verbo & suf. deverbativo \\
\hline
\end{tabular}

Elaboración propia.

El nombre del juego puede ser traducido como 'volcar y hacer que el otro vuelque algo'; pues el juego se desarrolla con dos participantes, donde el primero coloca el carro en el extremo inferior de una rampa y lo empuja con fuerza para que la recorra. La meta es que llegue sin ser volcado como se observa en la gráfico 1.

\section{Gráfico 1.}

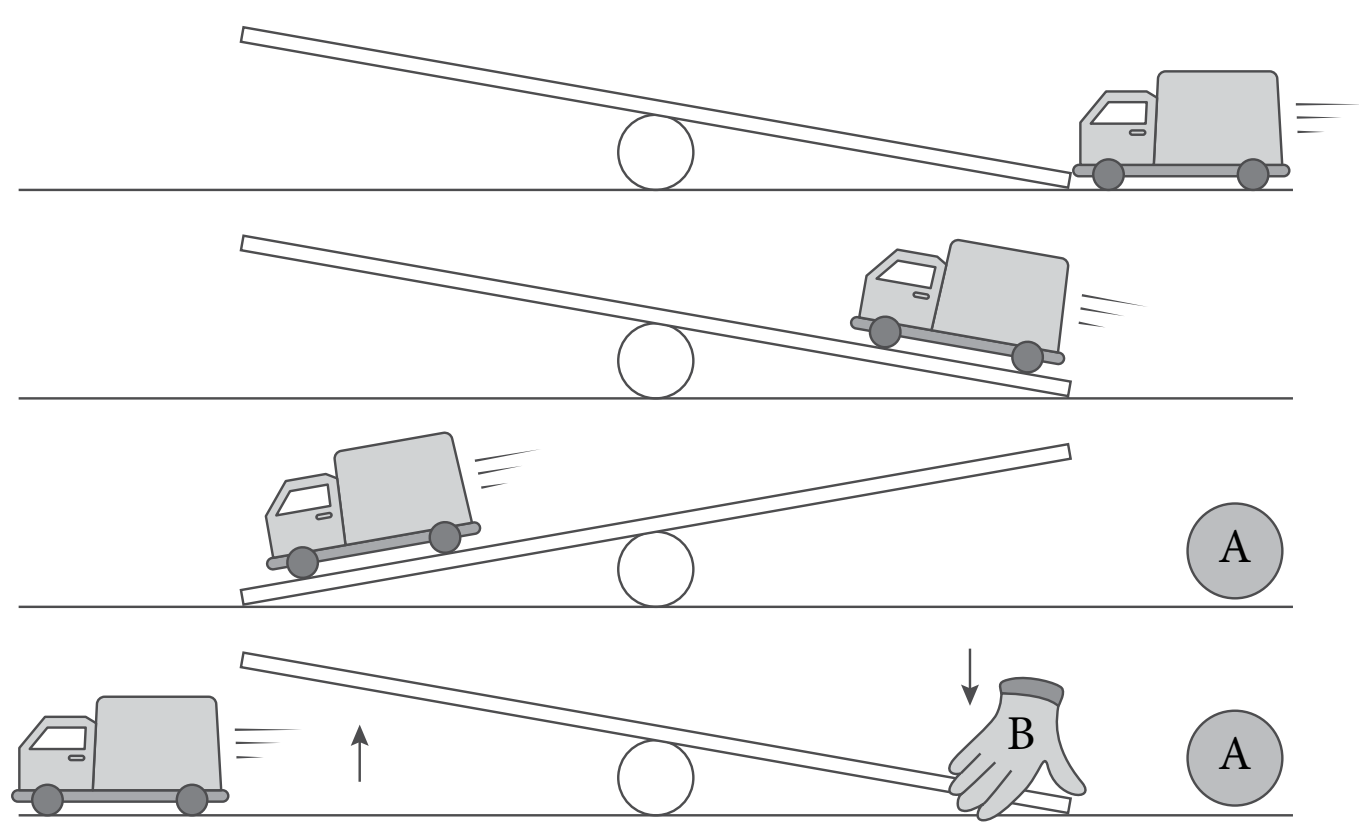

Elaboración propia. 
Si no se logra la meta anteriormente indicada, el jugador A pierde su turno y se lo pasa al jugador B. En este caso, el juego consiste en volcar el carro golpeando la parte posterior de la rampa, lo cual se ejemplifica en la gráfico 2.

\section{Gráfico 2.}

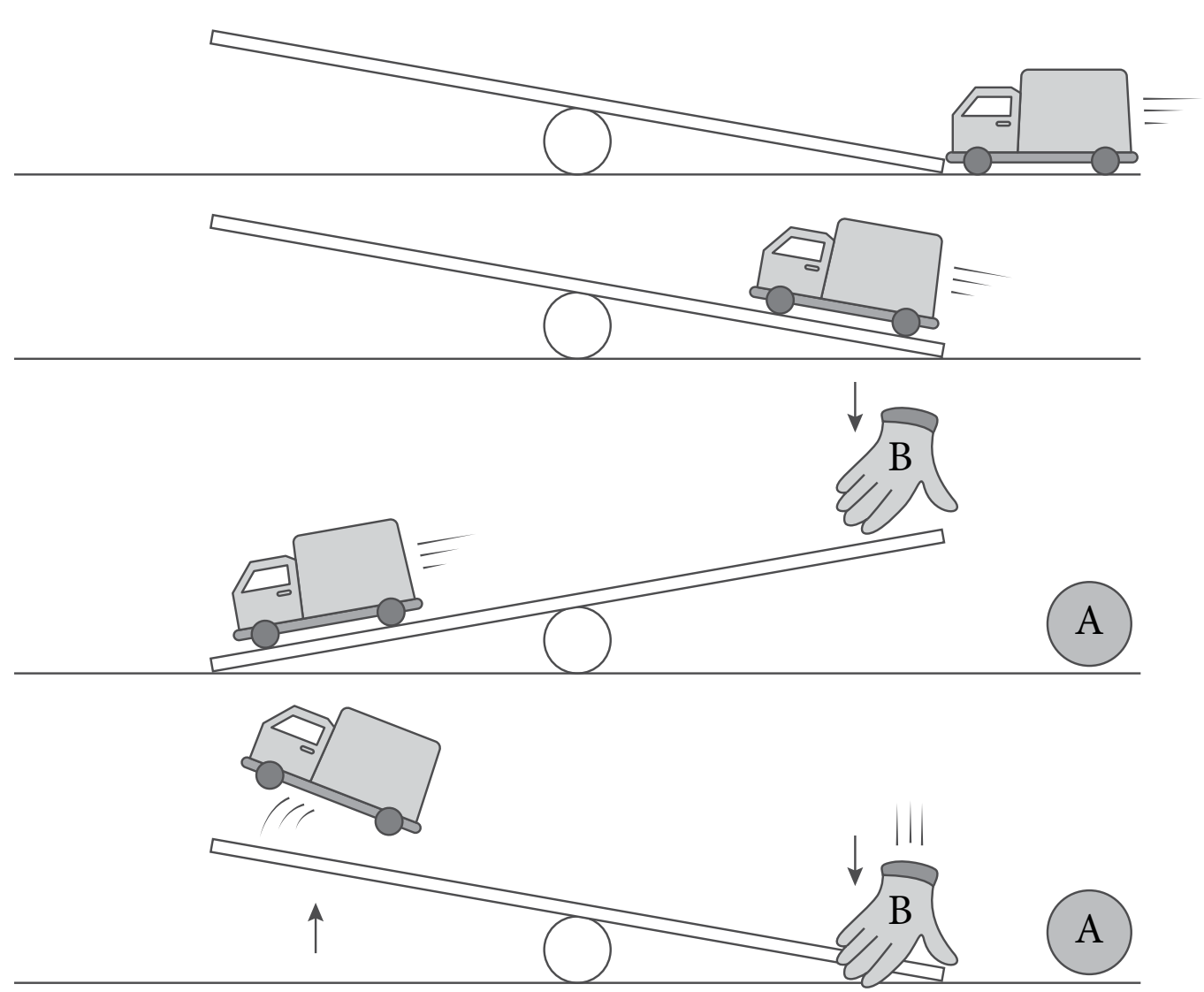

Elaboración propia.

El juego reproduce un ciclo, en donde se repite la acción, en este caso la de volcar. Martha Hardman, en Escobar (2017, p. 72), señala que la reduplicación «indica cantidad, énfasis y a veces metáfora. Aparece raras veces con verbos; ocurre más a menudo con sustantivos nominales y modificativos».Además, Hardman (en Escobar, loc. cit.) describe para la lengua jaqaru la siguiente clasificación: 
«tema $(\text { sin vocal })^{7}+$ tema + flexión. [...] La mayoría de las raíces nominalizadas y reduplicadas son topónimos y que la forma, en este caso es: raíz sin la vocal + raíz completa.»

A pesar de que en la presente investigación se trata de nominalizaciones de juegos, sucede que los topónimos son también nominalizaciones; entonces, es probable que tengan la misma estructura nominal para la formación de sustantivos, a pesar de que algunos de los juegos provienen de verbos. Escobar (ibídem: 73) brinda algunos ejemplos reduplicación en jaqaru:

Tabla 11.

\begin{tabular}{l|l}
\hline wala & v. correr \\
walwalara & v. huir en diferentes direcciones \\
\hline qala & s. piedra \\
qalqala & s. pedregal \\
\hline willu & n. curva \\
willwillu & n. zigzag \\
\hline
\end{tabular}

Extraído de El Jaqaru, último relicto aru en los Andes Centrales (2017, p. 73), de Emérita Escobar.

Para el JR con sufijo se observa que no es un sufijo de flexión, sino un derivativo, y la estructura es similar a como la presenta Martha Hardman (en Escobar, ibídem, p. 72).

Después de lo indicado, observamos que la reduplicación, en este caso, es un fenómeno que se relaciona con los mecanismos de innovación para la conceptualización y la cultura de los quechuahablantes.

Del JR volca volcachi se puede decir que expresa las funciones de [cantidad], [oscilación], [frecuencia] y [rapidez de la acción].

En contraste a la nominalización del JR volca volcachi, se tiene el JNR kallampi. La descripción morfológica de este nombre es la siguiente:

7 Al pertenecer a la familia lingüística Aru, lenguas como el aimara o el cauqui presentan el fenómeno de elisión vocálica, no encontrado en el quechua. 
Fernando Aarón Torres Castillo y Ana Priscila Ugarte de la Fuente

Tabla 12. Segmentación morfológica de Kallampi

\begin{tabular}{l|l|l}
\hline kalla /qala/ & $-\mathrm{m} /-\mathrm{mi}$ & -pi \\
\hline \multirow{2}{*}{ cuero } & $\begin{array}{l}\text { validador (también funciona como } \\
\text { el verbo ser si se encuentra en } \\
\text { tercera persona) }\end{array}$ & Locativo \\
\hline
\end{tabular}

El caso del JNR kallampi (lit. 'está en el cuero') es muy interesante, ya que presenta dos sufijos según Adelaar: el validador y el locativo. La palabra cuero aparece en la nominalización del JNR. Para entender mejor lo expuesto, se describen a continuación los componentes del juego.

Tabla 13. Descripción de las herramientas del juego Kallampi

\begin{tabular}{l|l}
$\begin{array}{l}\text { El cuero hace referencia a la llanta que se } \\
\text { utiliza para este juego, puesto que de ese } \\
\text { material está hecha. Por lo que se observa, } \\
\text { la identificación del objeto representa una } \\
\text { relación de insumo-producto. Un proceso } \\
\text { que resultó ser una metáfora. }\end{array}$ \\
\hline $\begin{array}{l}\text { Normalmente se utilizan ramas con dos } \\
\text { ramificaciones para que hagan contacto } \\
\text { con la llanta. }\end{array}$ \\
\hline
\end{tabular}

Elaboración propia

A continuación, en la gráfico 3, se muestra cómo se desarrolla el juego. 


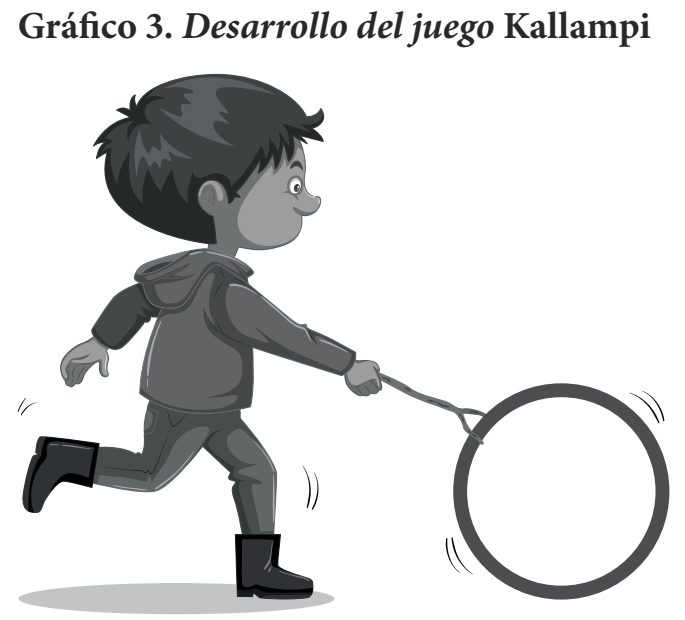

Elaboración propia

A diferencia del JR, en el JNR kallampi juega un solo participante; de acuerdo con ello, la falta de jugadores en este JNR sería una clara diferencia respecto de la función [cantidad] que se expresa en el primer JR con sufijo (volca volcachi). En este, necesariamente, se requiere de dos a más personas, a diferencia de kallampi donde el juego puede ser realizado por una sola persona. Aquello podría explicar por qué no hay reduplicación en el nombre del JNR kallampi, puesto que la función de [cantidad] no es necesaria para esta nominalización porque se trata de un solo jugador.

\subsubsection{Palabras reduplicadas sin sufijos}

A continuación, se desarrolla un análisis similar al anterior; pero en este caso los nombres de los juegos no presentan sufijos.

El JR Chilaq chilaq es un juego grupal y está sectorizado por categorías, estas categorías son personajes que los participantes escogen. Los personajes a escoger son tres: el lobo, la gallina y los pollitos. El juego consiste en que el lobo quiere llevarse a los pollitos, pero es interrumpido por la mamá gallina. En este caso, se plantea diferentes escenarios o posibles situaciones: si la gallina no llega a tiempo (ver gráfico 4), el lobo se lleva a uno de los pollitos; en el caso contrario, el lobo huye y no se lleva nada (ver gráfico 5). Este juego tiene una dinámica muy similar a la del juego San Miguel. 
Gráfico 4.
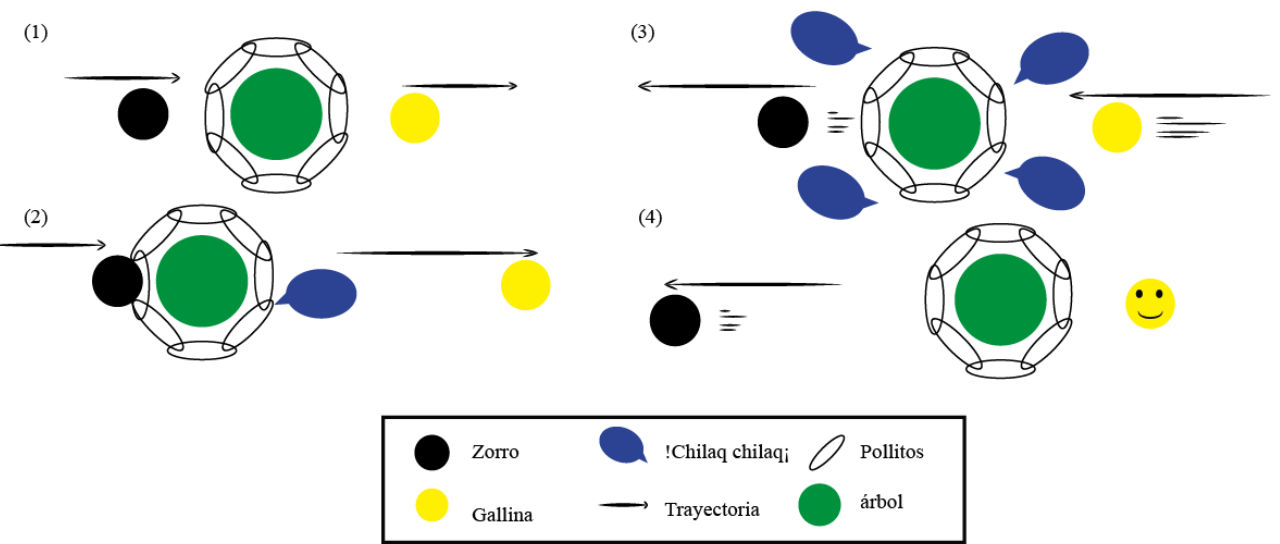

Elaboración propia

\section{Gráfico 5.}

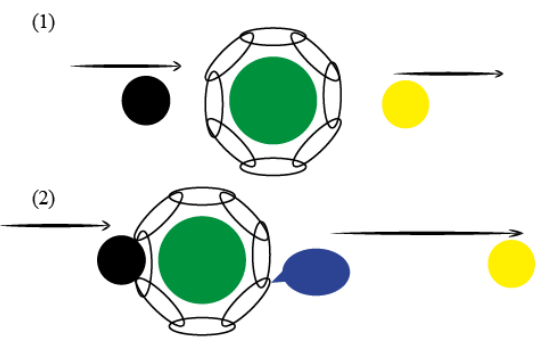

(3)

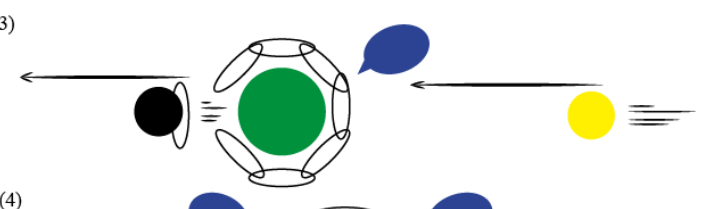

(4)

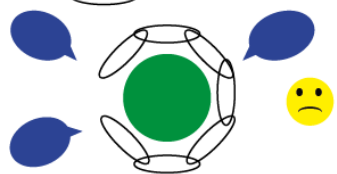

$$
\begin{aligned}
& \text { Zorro } \text { iChilaq chilaqi } O \text { Pollitos } \\
& \text { Gallina } \longrightarrow \text { Trayectoria } \bigcirc \text { árbol }
\end{aligned}
$$

Elaboración propia

Los niños que cumplen el papel de pollitos se encuentran alrededor del árbol, sujetados todos del antebrazo, y forma una cadena. Cuando el lobo se acerca y quiere llevarse a uno de ellos, todos comienzan a gritar ¡Chilaq chilaq!, como aviso para que el personaje que cumple el papel de la mamá gallina regrese y asuste al lobo para que este se vaya. 
$\mathrm{El}$ ¡Chilaq chilaq! hace referencia al sonido que emiten los pollitos en situación de peligro, lo que para un hispanohablante equivaldría a ipío pío!; pero en quechua la onomatopeya es chilaq chilaq. Este sonido onomatopéyico se ha nominalizado en el nombre de este JR.

En el siguiente gráfico se muestra la generalización, donde los sonidos repetidos se reducen a dos momentos para dar lugar a la nominalización.

\section{Gráfico 6.}

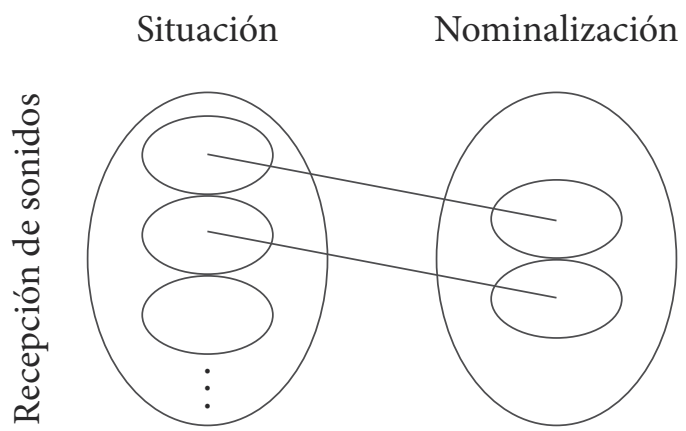

Elaboración propia

De esa forma se explicaría el nombre de juego Chilaq chilaq, donde un sonido que se repite periódicamente ha sido nominalizado. En el JR Chilaq chilaq se presentan las funciones de [cantidad], [frecuencia], [rapidez de la acción] y [oscilación].

Ahora se presenta el caso del JR paka paka ${ }^{8}$, el cual consiste en esconderse de una persona que busca a los jugadores que se han escondido. En Perú este juego es conocido también como «Las escondidas». En el diccionario elaborado por Durán (1967), la palabra pakay se define como 'ocultar'. El nombre del juego se puede estructurar de la siguiente forma:

Tabla 14. Segmentación morfológica del juego paka paka

\begin{tabular}{l|l}
\hline paka & paka \\
\hline ocultar & ocultar \\
\hline \multicolumn{2}{c}{ n. las escondidas } \\
\hline
\end{tabular}

8 Revisar RED dentro de ítemes léxicos: paka paka. 
En este JR se presentan las funciones de [cantidad], [frecuencia], [en varios puntos o espacios] y [rapidez de la acción].

Otro ejemplo es el JR chapa chapa, nombre que proviene del verbo español chapar. En el Diccionario de la lengua española (2014), el verbo chapar se define como 'agarrar'; esto para Perú y Uruguay.

La traducción del nombre es presentada literalmente como agarrar agarrar, puesto que no presenta ningún sufijo.

Tabla 15. Segmentación morfológica del juego chapa chapa

\begin{tabular}{l|l}
\hline Chapa & chapa \\
\hline Agarrar & agarrar \\
\hline \multicolumn{2}{c}{ n. las chapadas } \\
\hline
\end{tabular}

Al igual que en el juego anterior, este presenta las funciones de [cantidad], [frecuencia], [en varios puntos o espacios] y [rapidez de la acción].

Otro ejemplo es el JR pis pis, que entre los hablantes de castellano en Perú es conocido como yases. Este juego se originó en México, donde es llamado matatenas; su nombre deriva del náhuatl matatena 'llenar de piedras'.

Sin embargo, el nombre quechua para este juego no ha sido dado por las piezas del juego - que se llaman yases - , por lo que cabe preguntar de dónde proviene el nombre pis pis. En este caso se podría plantear que el nombre proviene de un sonido, el que se produce cuando chocan los yases, al cual los niños han identificado como [pis], el cual ha sido reduplicado por las funciones que presenta, que son las de [oscilación], [cantidad], [frecuencia], [en varios puntos o espacios] y [rapidez de la acción].

A continuación se presentan los JNR sin sufijos: taka y daño.

En el caso de taka, proviene del quechua takay 'golpear'. En Perú, este juego también se conoce como «trompo». Se plantea que el nombre de este juego procede de una de las técnicas que se usa en el desarrollo del mismo chantar (ver gráfico 7); esta acción es usada como castigo al jugador que pierde. 


\section{Gráfico 7.}
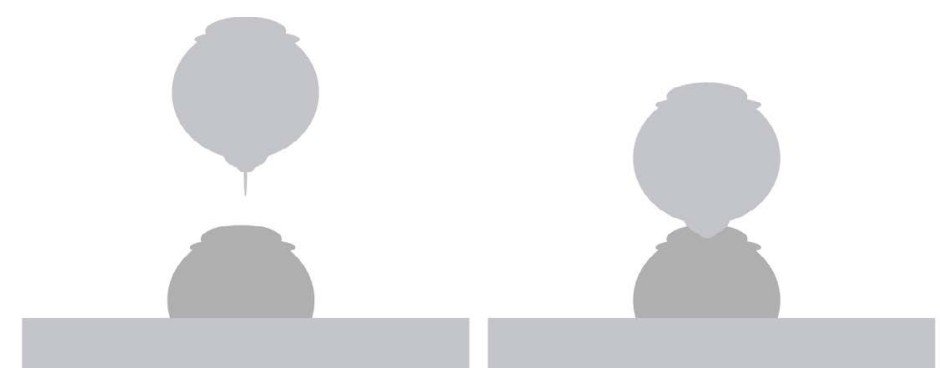

Elaboración propia.

Un juego con nombre similar a este, pero con reduplicación, es el taka taka, también conocido como el fulbito de mesa. Este no ha sido registrado como juego de los niños de Ayacucho; puesto que, sobre todo, lo juegan jóvenes, e incluso adultos. Este nombre también sería producto de una semejanza fonética por repetición del sonido takataka que se produce al jugar, como en el caso de ¡Chilaq chilaq!, donde sí se presentan las funciones de [cantidad], [frecuencia], [en varios puntos o espacios] ${ }^{9}$, [rapidez de la acción] y [oscilación], a diferencia de taka, donde no se presenta la función de [cantidad] y [frecuencia], pero sí las funciones de [en varios puntos o espacios] y [rapidez de la acción]. ¿Estas diferencias serán determinantes para que se manifieste el fenómeno de la reduplicación o al existir ya un JR con el mismo verbo se evita que se nominalice de igual forma? Por ello, hay que tener en cuenta algunas diferencias:

Tabla 16. Diferencia entre los juegos taka $y$ taka taka

\begin{tabular}{l|l}
\hline taka & taka taka \\
\hline $\begin{array}{l}\text { Se puede jugar solo o con varias } \\
\text { personas. }\end{array}$ & $\begin{array}{l}\text { Necesariamente se juega con } \\
\text { varias personas. }\end{array}$ \\
\hline $\begin{array}{l}\text { Cada jugador solamente tiene un } \\
\text { trompo. }\end{array}$ & $\begin{array}{l}\text { Cada jugador posee diferentes } \\
\text { jugadores en el tablero. }\end{array}$ \\
\hline Unidireccional & Multidireccional \\
\hline
\end{tabular}

Elaboración propia

9 Se explica al ver la distribución de golpes en la superficie del trompo que recibe la acción. 
Con respecto del JNR llamado daño, este nombre es español y hace referencia al juego de las canicas. La función que cumplen las canicas se ha convertido en el nombre asignado al juego; esta relación semántica de nombre y efecto del objeto se verifica también con el JNR taka (ver gráfico 8), ya que la función del juego taka es golpear el trompo del oponente para que pierda. En el caso del juego de las canicas, se busca «hacer daño» a la canica del oponente para eliminarlo del juego.

\section{Gráfico 8.}

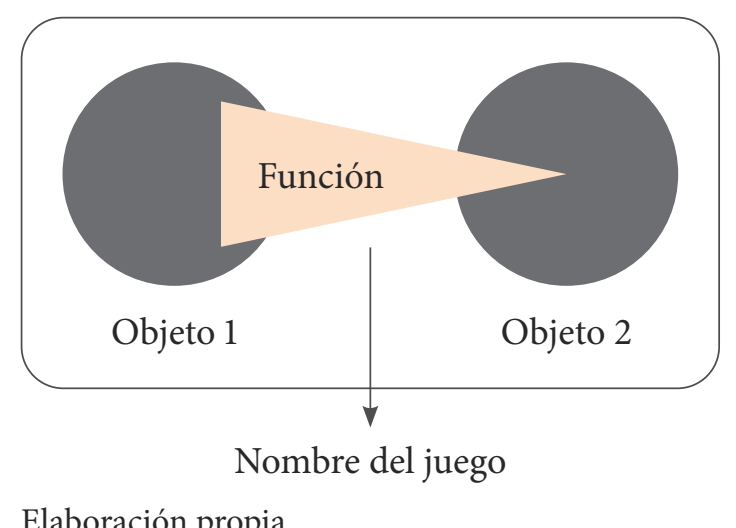

Elaboración propia.

En este JNR, daño, se presentan las funciones de [en varios puntos o espacios] y [rapidez de la acción].

\section{Resultados}

En la siguiente tabla se muestra qué juegos cumplen con las funciones propuestas.

Tabla 17. Distribución de las funciones semánticas según los juegos reduplicados

\begin{tabular}{l|l|l|l|l|l}
\cline { 2 - 6 } & [cantidad] & [frecuencia] & $\begin{array}{l}\text { [en varios } \\
\text { puntos o } \\
\text { espacios] }\end{array}$ & $\begin{array}{l}\text { [rapidez de } \\
\text { la acción] }\end{array}$ & [oscilación] \\
\hline Volca volcachi & $\mathrm{X}$ & $\mathrm{X}$ & & $\mathrm{X}$ & $\mathrm{X}$ \\
\hline Chilaq chilaq & $\mathrm{X}$ & $\mathrm{X}$ & & $\mathrm{X}$ & $\mathrm{X}$ \\
\hline Paka paka & $\mathrm{X}$ & $\mathrm{X}$ & $\mathrm{X}$ & $\mathrm{X}$ & \\
\hline
\end{tabular}




\begin{tabular}{|c|c|c|c|c|c|}
\hline & [cantidad] & [frecuencia] & $\begin{array}{l}\text { [en varios } \\
\text { puntos o } \\
\text { espacios] }\end{array}$ & $\begin{array}{l}\text { [rapidez de } \\
\text { la acción] }\end{array}$ & [oscilación] \\
\hline Chapa chapa & $\mathrm{X}$ & $\mathrm{X}$ & $\mathrm{X}$ & $\mathrm{X}$ & \\
\hline Pis pis & $\mathrm{X}$ & $\mathrm{X}$ & $\mathrm{X}$ & $\mathrm{X}$ & $\mathrm{X}$ \\
\hline Kallampi & & & $\mathrm{X}$ & $\mathrm{X}$ & \\
\hline Taka & & & $\mathrm{X}$ & $\mathrm{X}$ & \\
\hline Daño & & & $\mathrm{X}$ & $\mathrm{X}$ & \\
\hline
\end{tabular}

Elaboración propia.

\section{Conclusiones}

1. Las funciones de [cantidad], [frecuencia] y [rapidez de la acción] serían las más importantes para el fenómeno de la reduplicación en la nominalización de los juegos de los niños quechuahablantes.

2. Con respecto a la lengua quechua en su variación ayacuchana, existe similitud de reduplicación con sus variedades lingüísticas, asimismo con quienes comparte el mismo entorno geocultural.

3. De lo analizado, el verbo es la categoría reduplicada con más frecuencia en las nominaciones de los juegos de los niños hablantes del quechua ayacuchano.

\section{Referencias bibliográficas}

Casado, M. (1991). Lenguaje y cultura/ La etnolingüística. Madrid: Ariel.

Durand, H. (1967). Pequeño diccionario castellano quechua / quechua-castellano. Lima: Universo.

Escobar, E. (2017). El Jaqaru, último relicto aru en los Andes Centrales. Lima: Universidad Nacional Agraria La Molina.

Armendáriz, F. (2007). A Grammar of River Warihio (Tesis). Universidad de Rice, Estados Unidos.

Guerrero, L. (2014). ¿Sustantivos plurales, pluralia tantum o clases nominales? La codificación de plural en lenguas yutoaztecas. En R. Barriga y E. Herrera (Eds.), Estructura, cultura y hablantes (pp. 1-20). México: Colmex. 
Hualde, J., Ollarea, A., Escobar, M., Travis, C., y Sanz, C. (2001). Introducción a la Lingüística Hispánica. Edimburgo: Universidad de Cambridge.

Martinez, C. (1994). La reduplicación nominal en la lengua yaqui de Sonora. Estudios de Lingüística y Sociolingüística (pp. 157-178).

Ministerio de Educación. (2005). Yachakuqkunapa Shimi Qullqa. Lima: Autor.

Soto Ruiz, C. (2006). Quechua/ Manual de enseñanza. Lima: Instituto de Estudios Peruanos.

Weber, D. (1996). Una gramática del quechua del Huallaga (Huánuco). Lima: ILv. Zariquiey, R., y Córdova, G. (2008). Qayna, Kunan, Paqarin / Una introducción práctica al quechua chanca. Lima: PUCP. 